\title{
Workload-dependent queuing model of an AQM-controlled wireless router with TCP traffic and its application to PER-based link adaptation
}

\author{
Onur Ozturk and Nail Akar
}

\begin{abstract}
We propose a novel workload-dependent queuing model for a wireless router link which employs active queue management (AQM) and is offered with a number of persistent Transmission Control Protocol (TCP) flows. As opposed to existing work that focus only on the average queue occupancy as the performance metric of interest, the proposed analytical method obtains the more information-bearing steady-state queue occupancy distribution of the wireless AQM link. Simulations are performed to demonstrate the accuracy of the proposed model in both wireline and wireless scenarios. With the intention of maximizing TCP throughput, this analytical method is used to obtain guidelines for setting the target wireless packet error rate (PER) for a PER-based traffic-agnostic link adaptation scheme.
\end{abstract}

Keywords: Workload-dependent queues; TCP; Active queue management; Link adaptation; Cross-layer analysis

\section{Introduction}

Transmission Control Protocol (TCP), along with User Datagram Protocol (UDP), has been the most dominant transport protocol used in the Internet today. The traditional technique of using buffer management based on tail drop at wireline router links carrying TCP traffic leads to the so-called 'full queues' and 'lock-out' problems described in [1]. The full queues problem refers to the buffer being full most of the time, introducing large queuing delays which in turn impact adversely the TCP level throughput. The lock-out problem refers to a situation in which a single or a few flows monopolize the queue space while starving others as a result of synchronization or other timing effects. To avoid the full queues problem, active queue management (AQM) mechanisms drop packets before the queue becomes full [1]. Typically, the AQM drop decision is probabilistic on certain queue parameters to mitigate the lock-out problem [1]. For various AQM mechanisms proposed in the literature, we refer the reader to [1-5]. More recently, Adams [6]

*Correspondence: ozturk@ee.bilkent.edu.tr

Electrical and Electronics Engineering Department, Bilkent University, Bilkent, Ankara 06800, Turkey presents a comprehensive survey of AQM along with an elaborate classification and comparison of its proposed variants and its use in the wireless context. Lakkakorpi et al. [7] conclude that the standards-based Worldwide Interoperability for Microwave Access (WiMAX) technology can indeed benefit from $\mathrm{AQM}$ in reducing its downlink latency, and we use the WiMAX physical layer in the numerical experiments of this paper.

An analytical expression, the so-called PFTK formula, is provided for the steady-state throughput of a persistent or long-lived TCP flow (i.e., a flow with a large amount of data to send such as FTP transfers) as a function of its packet loss rate and the round-trip time (RTT) in [8]. The PFTK formula takes into account both the fast retransmit mechanism of TCP Reno and the effect of TCP timeout on throughput. For a related study on a simpler TCP throughput expression which ignores certain features of TCP, the so-called square-root formula, see [9]. Lassila et al. [10] further integrate the square-root model with a generalized processor sharing model to analyze nonpersistent TCP flows as well. Using fixed-point iterations, the PFTK formula can be used to approximate the absolute throughput of a TCP flow sharing an AQM router

\section{Springer}

(c) 2014 Ozturk and Akar; licensee Springer. This is an Open Access article distributed under the terms of the Creative Commons Attribution License (http://creativecommons.org/licenses/by/2.0), which permits unrestricted use, distribution, and reproduction in any medium, provided the original work is properly credited. 
link with other TCP flows and also in a network of AQM routers with persistent and dynamic traffic scenarios [11]. However, the focus in [11] is the mean queue occupancy in router links, not the queue occupancy distribution. For related work on how to approximate the flow-level TCP throughput when the flows share a network of AQM routers using an $\mathrm{M} / \mathrm{M} / 1 / \mathrm{K}$ model, we also refer the reader to [12].

In wireline router links, errors due to transmission are negligible. However, in wireless router links, packet errors due to transmission are inevitable. Therefore, in addition to congestion losses stemming from AQM drops, we also have non-congestion (or wireless) losses arising due to channel errors. TCP suffers substantially from non-congestion losses since it responds to all losses by invoking congestion control and avoidance algorithms which results in degraded end-to-end performance on paths with lossy links [13]. A comparison of different approaches for improving TCP performance over wireless links is provided in [13]. Barakat and Altman [14] use the square-root formula to analytically study the interaction between TCP and the amount of forward error correction (FEC) to be used in wireless links. This study is then extended to the interaction of TCP and automatic repeat request with selective repeat (ARQ-SR), and inorder delivery of packets to the IP layer, using the PFTK formula. Optimal design and analysis of hybrid FEC/ARQ schemes in TCP context is also studied in [15] and [16] with Rayleigh fading. In these studies, the AQM mechanism is not taken into consideration. A recent work in [17] studies an AQM-controlled wireless link with FEC and ARQ using fixed-point approximations and the PFTK formula in an $\mathrm{M} / \mathrm{M} / \mathrm{1} / \mathrm{K}$ setting. One of the goals of this paper is to introduce a novel queuing model for AQMcontrolled wireless links with the specific goal of obtaining the steady-state queue occupancy distribution.

While AQM focuses on buffer management addressing the full queues and lock-out problems, link adaptation (LA) adapts the transmission parameters of the wireless system to changing channel conditions with the intention of increasing the spectral efficiency of the wireless transmission system [18]. The key parameters to adapt are the modulation and coding levels also known as adaptive modulation and coding (AMC), transmission power, spreading factors, etc., or a hybrid of the above. One of the topics we study in the current paper is AMC-based LA without power control for which in real implementations, the values for the transmission parameters are quantized and grouped together into a finite set of modulation and coding schemes (MCSs). The basic goal of an LA algorithm is to choose the best possible MCS over varying channel conditions [18], whereas the main principle is to use in this decision a certain channel state information (CSI) representative of the quality of the wireless channel. CSI may be in the form of SNR or signal-tonoise-plus-interference ratio (SINR) that is available from the physical layer [18]. Additionally, the wireless channel is assumed to match a particular stochastic model, whichever suits, to indirectly obtain packet error rate (PER) which is crucial for MCS selection [19]. Mapping estimated SNR values to MCSs, however, presents a challenge in multipath fading channels for which the performance of a given MCS of interest may exhibit significant variation across different channel models. It is shown in [20] and [21] that the average throughput can be significantly increased if the MCS selection is based on an accurate prediction of PER to be expected for the current channel conditions. However, to obtain a reliable estimate of PER, a large number of packets need to be transmitted, making the adaptation relatively slower when compared to SNR-based LA [18].

In this paper, we study a wireless link which can be viewed as the downlink of a cellular wireless network comprising a base station (BS) serving a number of mobile stations (MSs) or a wireless link between two routers. We assume that the wireless link employs AQM and is offered with a fixed number $N$ of long-lived TCP Reno flows in one direction, all using a fixed packet size $L$. TCP ACK packets are transmitted in the other direction with priority given to ACK traffic so that delay and losses for ACK traffic can safely be neglected. For the downlink scenario, data flows from the network to MSs which send their ACK packets in the other direction and the uplink data traffic is not considered. Such wireless links are categorized as infrastructure type in [6], and they are identified as potential bottleneck links due to possible bandwidth mismatch between the wireless and wired domains. In accordance with this observation, we assume in this paper that all flows are either bottlenecked at this particular link or the bottleneck bandwidth of the flows are fixed and known in advance. Propagation delays, fixed part of the RTT, of individual flows are allowed to be arbitrary. An MCS is used to serve the packets waiting in the queue, and errored packets are not retransmitted, i.e, ARQ or hybrid ARQ (HARQ) mechanisms are not in action. We address the PER-based LA problem of choosing the best possible MCS to maximize the total throughput of TCP flows. PER-based LA comprises the following three components: (i) PER estimation, (ii) determination of the target PER or a range of target PERs, and (iii) the LA algorithm. In this paper, we address the second component and we study the target PER that needs to be maintained by a PER-based LA algorithm to maximize the overall throughput of the TCP flows that share the wireless link. To explain, a low target PER leads to a situation in which losses are mostly due to AQM drops, but since the service rate of the queue would be relatively limited to achieve a low PER, reduced TCP level throughput is inevitable. On the other hand, a 
high target PER increases the queue service rate, but it becomes quite possible that the queue would occasionally be empty due to substantial wireless losses stemming from TCP reaction to such losses. Note that TCP throughput optimization is shown to be dependent not only on PER but also on traffic-related parameters such as the number of TCP flows and their RTT values. However, estimating the number of active TCP connections and their RTT values is computationally difficult to implement. In this paper, we study only traffic-agnostic PER-based LA.

The two main contributions of this paper are presented below:

- We introduce a novel workload-dependent M/D/1 queuing model for an AQM-controlled wireless link with Bernoulli packet losses using the PFTK formula taking into account both the fast retransmit mechanism of TCP Reno and the effect of TCP timeout on throughput to obtain the entire queue occupancy distribution. In most existing work, the focus has been on the mean queue occupancy as well as the average packet loss probability due to congestion, and moreover, fixed packet sizes are generally not taken into account. The proposed model is validated by ns-3 [22] simulations. The following are the main features of the proposed model: (i) The proposed model provides a good match with simulations even in the vicinity of empty queues as opposed to other existing models. The 'empty queues' scenario is particularly important when an MCS is used with high wireless loss rates, and TCP sources throttle back relatively aggressively in a way that they cannot keep the queue full all the time. (ii) Some of existing models suffer when the workload-dependent AQM packet drop probability is discontinuous with respect to the workload, whereas the performance of our proposed method is insensitive to such behavior. (iii) The proposed method can further be used in the analysis of quality of service (QoS) differentiation mechanisms relying on per-class buffer management such as weighted random early detection (WRED) [23,24].

- We present a novel cross-layer framework based on the proposed queuing model to obtain a range of target PERs that needs to be maintained by a traffic-agnostic PER-based LA scheme for TCP throughput optimization. By being traffic-agnostic, optimality is shown to be sacrificed but the proposed range of target PERs allows one to obtain robust TCP performance for a wide range of traffic parameters including the number of TCP flows, their RTTs, etc. In this description, a robust policy refers to one that does not deviate much from an optimal policy that requires a priori information about the underlying traffic parameters. For the cross-layer framework, we use the IEEE 802.16e Wireless-Metropolitan Area Network (MAN) Orthogonal Frequency Division Multiplexing Access (OFDMA) Physical (PHY) air interface as the underlying PHY layer technology, but the framework allows other technologies to be used [25].

HARQ/ARQ techniques for which the errored packets at the receiver are retransmitted by the transmitter until either they are successfully decoded or a retransmission limit is reached are not considered in this paper. From TCP perspective, they prove to be powerful techniques to combat with multipath fading in wireless channels at the expense of increased delay and jitter caused by random retransmissions. Out-of-order packet delivery is another natural consequence of HARQ/ARQ which can be detrimental to TCP throughput if left uncompensated at the receiver [26]. Out-of-order packets force TCP receivers to send duplicate ACKs to TCP transmitters as if the missing packets have been lost which in turn throttles back the transmitter's packet injection rate to the network. To cope with out-of-order packet arrivals, wireless receivers optionally resequence the arriving packets from the air interface before their delivery to the network [27]. For the analysis of TCP performance over links deploying HARQ/ARQ, one should additionally model the complex effects of the retransmission delays as well as resequencing delays. TCP modeling for AQM-controlled links with HARQ/ARQ is left for future research.

The paper is organized as follows. In Section 2, the workload-dependent queuing model for an AQMcontrolled wireless link with Bernoulli wireless packet losses is presented. In Section 3, the model is validated in both wireline and wireless scenarios using simulations. Section 4 addresses the framework we introduce using the proposed queuing model to obtain the target PER for TCP throughput optimization for a wide range of scenarios. We conclude in the final section.

\section{Analytical model}

\subsection{Workload-dependent M/G/1 queue}

The following description of workload-dependent M/G/1 queues and the accompanying notation is based on [28]. We consider a Markovian workload process in which the server drains the queue according to a workloaddependent service rate function $r(x)$ (in units of bps) where $x$ denotes the current workload (in units of bits) in the queue. On the other hand, the packet arrivals to the queue are governed by a Poisson process with a workload-dependent intensity function $\lambda(x)$. We assume $r(0)=0, r(x)$ is strictly positive, leftcontinuous, and has a strictly positive right limit on $(0, \infty)$. Each arrival increases the workload by the 
job size (in units of bits) whose cumulative distribution function (CDF) is denoted by $B(\cdot)$ with mean job size $\beta$. Under the condition $\limsup _{x \rightarrow \infty} \beta \frac{\lambda(x)}{r(x)}<1$, the workload process is ergodic and possesses a stationary probability density function (PDF) denoted by $v(x), x>0$. The workload process may also have a probability mass (atom) at zero denoted by $V(0)$. It is shown in [28] that the steady-state workload density $v(\cdot)$ satisfies the following integro-differential equation for $x>0$ :

$$
\begin{aligned}
r(x) v(x)= & \lambda(0) V(0)(1-B(x)) \\
& +\int_{y=0^{+}}^{x}(1-B(x-y)) \lambda(y) v(y) \mathrm{d} y,
\end{aligned}
$$

where the function $\tilde{R}(x)$ defined as $\int_{0}^{x \lambda(y)} \mathrm{d} y$ and in case $\tilde{R}(x)<\infty, 0<x<\infty$, then (1) has a non-zero atom $V(0)$ at $x=0$. A closed form solution to (1) for the workloaddependent $M / M / 1$ queue is given in [28]. For persistent TCP flows, packet sizes are generally fixed rather than being variable for a given flow. Therefore, the specific case of the workload-dependent $\mathrm{M} / \mathrm{D} / 1$ queue and a numerical solution for finding the stationary density $v(x)$ is crucial for TCP modeling which is described next. For this purpose, we fix the deterministic packet length to $L$ bits. The buffer occupancy is then discretized with a discretization interval $\Delta$ such that $l=L / \Delta>>1$ is an integer. We then define $v_{i}=v(i \Delta)$ for $i>0$ and discretize the integro-differential equation (1) to obtain

$$
v_{i}= \begin{cases}\frac{\lambda_{0} V(0)+\sum_{j=1}^{i-1} \lambda_{j} v_{j} \Delta}{r_{i}-\lambda_{i} \Delta}, & i<l . \\ \frac{\sum_{j=i-l+1}^{i-1} \lambda_{j} v_{j} \Delta}{r_{i}-\lambda_{i} \Delta}, & \text { otherwise. }\end{cases}
$$

Note that the identity (2) enables us to calculate $v_{i}$ as a weighted sum of $v_{j}^{\prime}$ 's for $j<i$ which lends itself to an iterative procedure. We propose to set $V(0)=1$ and iteratively calculate $v_{i}$ for $1 \leq i \leq K$ as in (2) for some large choice of $K$. Note that $K$ should be chosen such that $\sum_{K+1}^{\infty} v_{i} \Delta$ should be small enough to yield an acceptable approximation error. Finally, we first define $V=V(0)+\sum_{i=1}^{K} v_{i} \Delta$ and then normalize as follows:

$$
V(0):=\frac{V(0)}{V}, v_{i}:=\frac{v_{i}}{V}, \quad 1 \leq i \leq K .
$$

\subsection{Equation-based TCP model}

In line with the majority of the existing work on TCP modeling, we propose to use the so-called PFTK TCP model of [8] to relate the throughput of a TCP flow to the packet loss rate seen by the flow. For details, we refer the reader to [8]. Let $p, \lambda$, and $T_{0}$ denote the packet loss rate, packet send rate, and the retransmission timeout parameter of a TCP source, respectively. In our model, we use the following relationship used in the implementation of TCP in [22]:

$$
T_{0}=\max \left(T_{0, \min }, \mathrm{RTT}+4 \sigma_{\mathrm{RTT}}\right),
$$

where RTT and $\sigma_{\mathrm{RTT}}$ are the smoothed estimates for the round-trip time (RTT) and its standard deviation, respectively, and $T_{0, \min }$ is a minimum limit imposed on the timeout parameter. Let $W_{u}$ denote the random variable associated with the unconstrained window size of the TCP source. Also, let $W_{\max }=W / L$ and $b$ denote the maximum window size in units of packets and the number of packets to wait before sending a cumulative ACK packet by the TCP receiver, respectively, where $W$ is the receiver's buffer size. Padhye et al. [8] propose the following equation for the TCP send rate $\lambda$ if the TCP flow is exposed to a packet loss rate of $p$ :

$\lambda= \begin{cases}\frac{\frac{1-p}{p}+E\left[W_{u}\right]+\tilde{Q}\left(E\left[W_{u}\right]\right) \frac{1}{1-p}}{\operatorname{RTT}\left(\frac{b}{2} E\left[W_{u}\right]+1\right)+\tilde{Q}\left(E\left[W_{u}\right]\right) T_{0} \frac{f(p)}{1-p}}, & E\left[W_{u}\right]<W_{\max } \\ \frac{\frac{1-p}{p}+W_{\max }+\tilde{Q}\left(W_{\max }\right) \frac{1}{1-p}}{\operatorname{RTT}\left(\frac{b}{8} W_{\max }+\frac{1-p}{p W_{\max }}+2\right)+\tilde{Q}\left(W_{\max }\right) T_{0} \frac{f(p)}{1-p}}, & \text { otherwise }\end{cases}$

where

$$
\begin{gathered}
f(p)=1+p+2 p^{2}+4 p^{3}+8 p^{4}+16 p^{5}+32 p^{6}, \\
\tilde{Q}(w)=\min \left(1, \frac{\left(1-(1-p)^{3}\right)\left(1+(1-p)^{3}\right)\left(1-(1-p)^{(w-3)}\right)}{1-(1-p)^{w}}\right),
\end{gathered}
$$

and

$$
E\left[W_{u}\right]=\frac{2+b}{3 b}+\sqrt{\frac{8(1-p)}{3 b p}+\left(\frac{2+b}{3 b}\right)^{2}} .
$$

Throughout our numerical studies, we fix $T_{0, \min }=0.2 \mathrm{~s}$ as in [29], $b=2, L=1,500$ bytes (unless otherwise stated), and $W=64$ Kbytes. With these, the equation (5) provides a closed-form expression for the TCP send rate $\lambda$ in terms of $p$, RTT, and $\sigma_{\mathrm{RTT}}$.

\subsection{Active queue management}

Active queue management (AQM) refers to a set of buffer management disciplines that are used in routers by which packets are dropped long before the queue reaches its full capacity [5]. AQM disciplines maintain a shorter average queue length than their drop tail counterparts which drop packets only when the queue capacity is full. Moreover, typical AQM schemes probabilistically drop packets to mitigate synchronization of TCP sources sharing the link. One of the pioneering AQM schemes is random early detection (RED) [2] for which an arriving packet is probabilistically dropped as a function of the average queue occupancy that is obtained by applying an autoregressive filter to the queue occupancy time series data. The packet drop rate of RED scheme is linear with respect to the average queue occupancy in a certain regime of the 
queue defined by certain thresholds. Performance of RED is known to exhibit considerable variation with respect to the particular choices for these thresholds [30]. As a remedy, the so-called gentle variant of RED, called GRED, is proposed to make RED less sensitive to the choice of these parameters [31]. Moreover, stochastic modeling of the autoregressive filtering operation used in such RED-like schemes is generally known to be difficult and costly $[32,33]$. We therefore propose to use early random detection (ERD) [3] for which an arriving packet is dropped with probability $p(x)$ when the instantaneous queue length takes the value $x$. The gentle variant of the ERD discipline we study in this paper refers to the particular choice of $p(x)$ as follows:

$p(x)= \begin{cases}0, & 0 \leq x<t h_{\min } \\ \frac{x-t h_{\min }}{t h_{\max }-t h_{\min }} p_{\max }, & t h_{\min } \leq x<t h_{\max } \\ p_{\max }+\frac{x-t h_{\max }}{t h_{\max }}\left(1-p_{\max }\right), & t h_{\max } \leq x<2 t h_{\max } \\ 1, & \text { otherwise. }\end{cases}$

In this paper, $t h_{\min }$ and $t h_{\max }$ are set to $30 L$ and $90 L$, respectively, in units of bits, and $p_{\max }$ is set to 0.1 .

\subsection{Proposed model}

We envision a wireless router link that is offered with persistent TCP flows with the following assumptions:

- $N$ persistent TCP flows share the wireless link using first-in-first-out (FIFO) queuing.

- All flows use the same packet size $L$.

- Incoming packets from the TCP flows are dropped according to the specific ERD AQM scheme given in (9) with the drop decision depending solely on the instantaneous queue occupancy $x$.

- The link serves the waiting packets in the queue with a fixed transmission rate $r_{m}$ dictated by the underlying MCS. We assume $M$ different physical layer MCSs denoted by $\mathrm{MCS}_{m}, m \in\{0, \ldots, M-1\}$ that are supported by the link's air interface. For this study, we will be given a fixed $\mathrm{MCS}_{m}$ and SNR level denoted by $\mathrm{SNR}_{\mathrm{s}}, s \in\{0, \ldots, S-1\}$, and we will assume that transmitted packets are errored at the receiver with a probability denoted by $\mathrm{PER}_{m, s}$. Lost packets are not retransmitted, and loss events are assumed to be independent and identically distributed (iid) following the Bernoulli wireless loss model.

- Flow $i, i \in\{0, \ldots, N-1\}$ is exposed to

- an average packet loss probability $\overline{p_{i}}$ accounting for losses generated by both the ERD scheme and wireless transmission.

- an instantaneous workload-dependent queuing delay $D(x)=x / r_{m}$ at the router when the instantaneous queue occupancy takes the value $x$. Without loss of generality, $D_{T}=L / r_{m}$ and $D_{F}$ account for the transmission and one-way framing delays, respectively, the latter including other processing delays of any wireless communication system. $D_{F}$ is multiplied by a factor of 2 to account for both forward and reverse (TCP ACK messages) path delays.

- Queuing and transmission delays as well as the error rates of the TCP ACK packets are assumed to be negligibly small and will be ignored by the analytical model assuming that TCP ACK prioritization is deployed [34] and enhanced wireless protection is established in the reverse path of the flows [35].

- In addition to the delay encountered at the router, each flow has an additional fixed round-trip delay denoted by $\mathrm{RTT}_{0, i}, i \in\{0, . ., N-1\}$ taking into account the propagation delays of all links on the path of the flow.

- We assume that either all flows are bottlenecked at the particular wireless link of interest or their bottleneck bandwidths at other links are known in advance and they remain unaffected by the dynamics of the wireless link. Packet error rates on other links are assumed to be negligible. We therefore do not attempt to model networks of AQM router links but rather focus on a single AQM link.

- The packet send process for each flow is Poisson with intensity $\lambda_{i}$ which will be shown to depend on the instantaneous queue occupancy $x$ in our proposed model. We note that the Poisson assumption has successfully been used for TCP modeling in previous studies [17].

- All TCP flows use the same minimum timeout parameter $T_{0, \text { min }}$.

The central idea of this paper is to use the PFTK TCP model given in (5) to write $\lambda_{i}$ as a function of the single independent parameter $x$ so that the stationary queue density can be obtained by solving the workloaddependent M/D/1 queue described in Section 2.1. For this purpose, let the workload-dependent round-trip delay of flow $i$ be denoted by $\operatorname{RTT}_{i}(x)$ which can be expressed as the sum of the following components:

$$
\mathrm{RTT}_{i}(x)=\mathrm{RTT}_{0, i}+2 D_{F}+L / r_{m}+x / r_{m} .
$$

Furthermore, let $T_{0, i}(x)$ denote the workload-dependent timeout parameter for flow $i$ which can be expressed via (4) as

$$
T_{0, i}(x)=\max \left(T_{0, \min }, \mathrm{RTT}_{i}(x)+4 \sigma_{\mathrm{RTT}, i}\right),
$$

where $\sigma_{\mathrm{RTT}, i}$ stands for the standard deviation of the router queuing delay for flow $i$. The overall rate of packets 

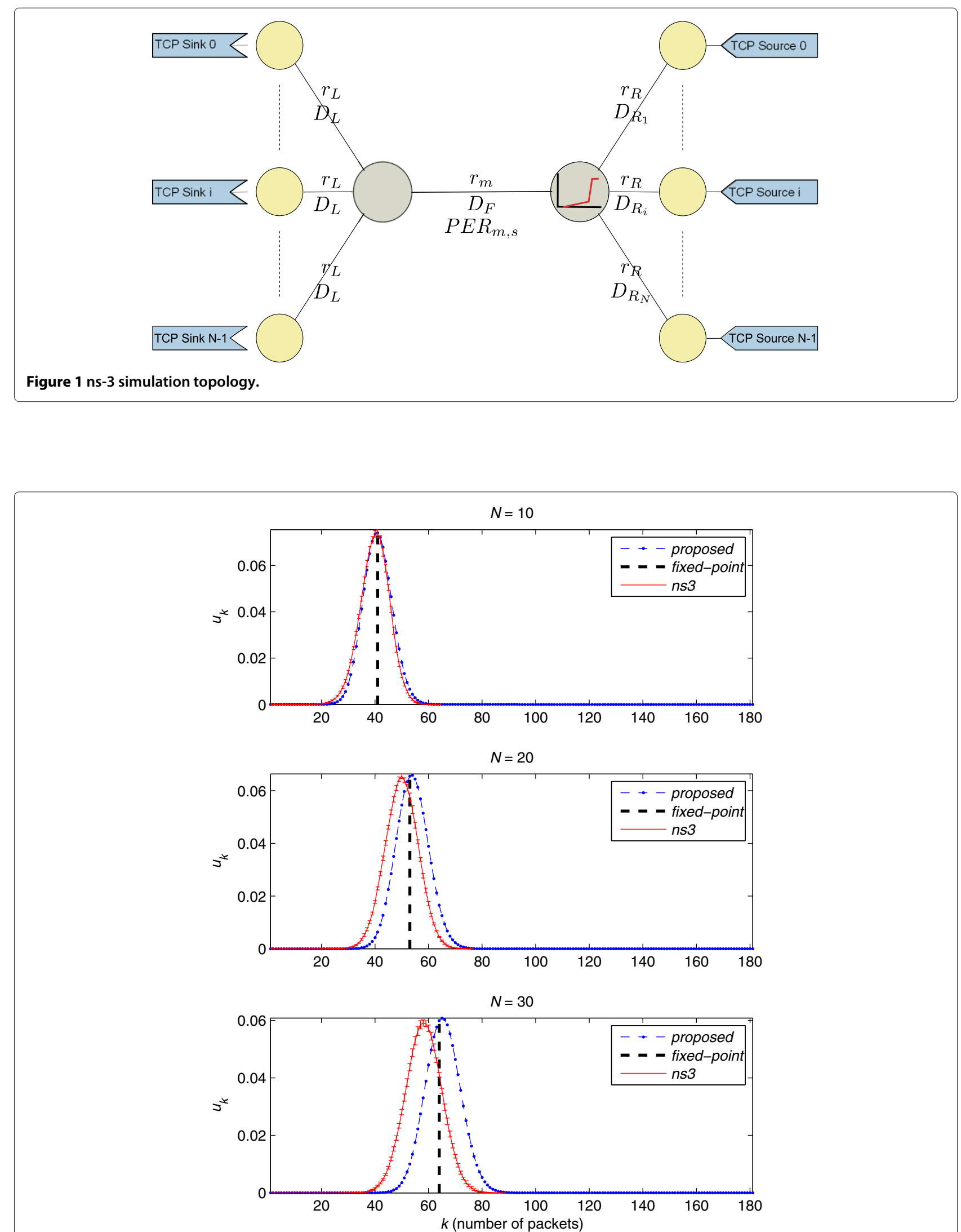

Figure 2 Queue occupancy PMF $u_{k}$ for three values of $N$ for scenario $A:\left(D_{L}, D_{F}, D_{R_{i}}\right)=(5,0,5) \mathrm{ms}$. 
that are admitted into the queue denoted by $\lambda(x)$ can then be written as

$$
\lambda(x)=\sum_{i=0}^{N-1} \kappa_{i}(x),
$$

where

$$
\kappa_{i}(x)=(1-p(x)) \lambda_{i}(x),
$$

is the rate of packets belonging to individual flow $i$ that is admitted into the queue. In our proposed model, $\lambda_{i}(x)$ is the send rate of flow $i$ when the queue occupancy takes the value $x$, and we propose to use the PFTK TCP model (5) to write $\lambda_{i}(x)$ with RTT and $T_{0}$ being replaced with their per-flow based workload-dependent counterparts $\mathrm{RTT}_{i}(x)$ and $T_{0, i}(x)$, respectively, and $p$ being replaced with its per-flow counterpart $\overline{p_{i}}$ which is the average packet loss probability for flow $i$. We put $\lambda_{i, \max }$ as an upper bound on $\lambda_{i}(x)$ representing the maximum send rate imposed on flow $i$ by the links it traverses other than the one under analysis. $\lambda_{i \text {,max }}$ is assumed to be known and set to $\infty$ throughout this paper unless otherwise stated. We note that the delay terms in (5) are taken as a function of the instantaneous queue occupancy $x$, whereas the loss probability term is obtained by averaging out over all possible values of $x$. We also studied alternative formulations for which the loss probability term is allowed to depend on $x$ which produced much less favorable results and are therefore not given in this study. We plug (12) into (1) and employ $B(x)=1$ for $x \geq L$ and zero otherwise. Moreover, for a given $\mathrm{MCS}_{m}$, we set $r(x)=r_{m}$ which then leads us to the framework of the workload-dependent M/D/1 queuing with fixed service speed. Since $p(x)=1$ and thus $\lambda(x)=0$ for $x \geq 2 t h_{\max }$ bits, the condition on $\tilde{R}(x)$ for a stationary solution $v(x)$ with an atom at zero can be shown to be satisfied for this particular model. Since the stationary density $v(x)=0$ for $x>x_{\max }=\left(2 t h_{\max }+L\right)$, we set $K=x_{\max } / \Delta$ in our numerical examples where $\Delta=1$ bit unless otherwise stated.
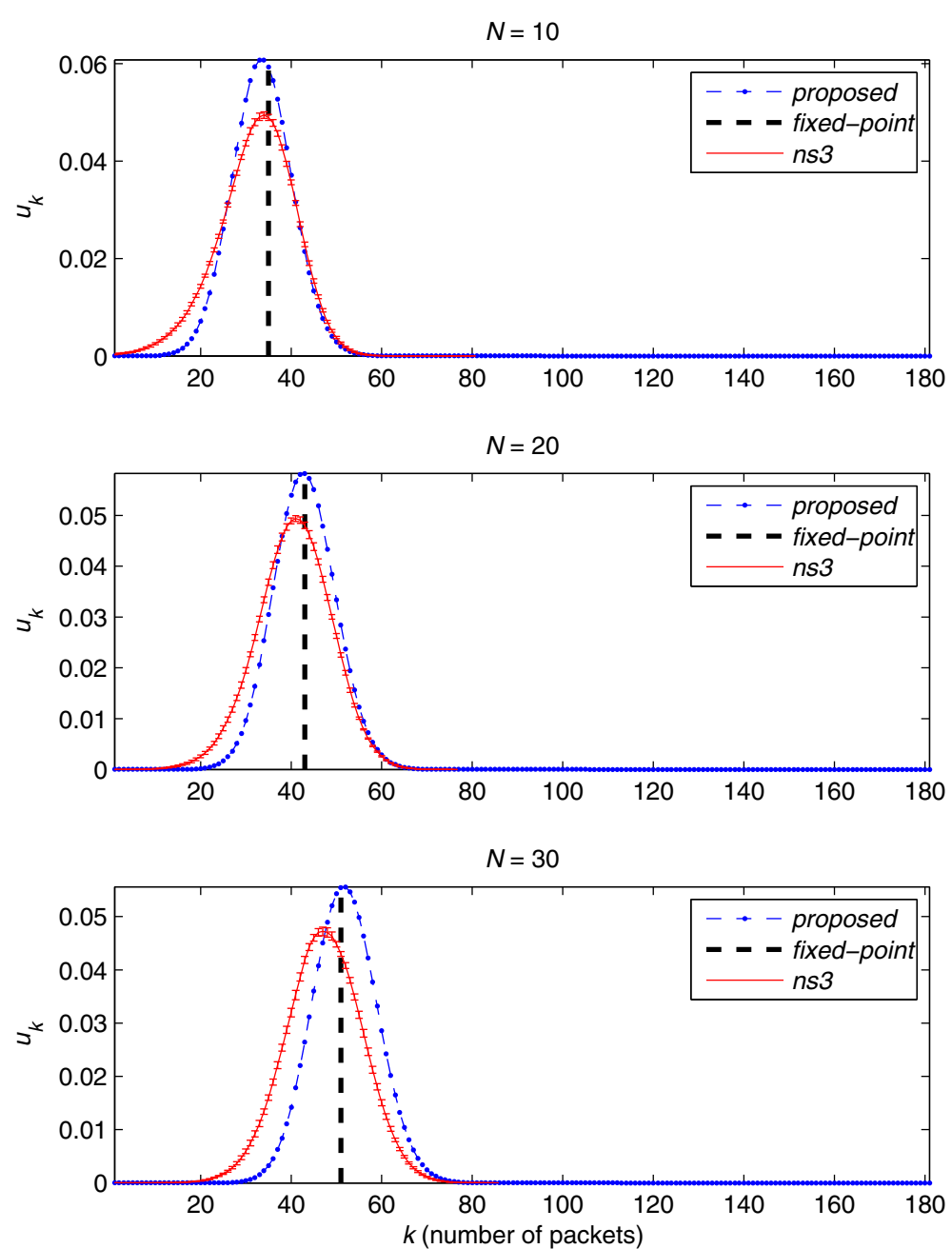

Figure 3 Queue occupancy PMF $u_{k}$ for three values of $N$ for scenario B: $\left(D_{L}, D_{F}, D_{R_{i}}\right)=(15,0,5+5\lfloor i /(N / 10)\rfloor) \mathrm{ms}$. 
Let $\overline{\lambda_{i}}$ and $\overline{\kappa_{i}}$ denote the average packet arrival and acceptance rates, respectively, into the queue from flow $i$. We can then write by queue-averaging arguments the following two identities:

$$
\begin{aligned}
& \overline{\lambda_{i}}=V(0) \lambda_{i}(0)+\int_{0^{+}}^{x_{\max }} \lambda_{i}(x) v(x) \mathrm{d} x, \\
& \overline{\kappa_{i}}=V(0) \kappa_{i}(0)+\int_{0^{+}}^{x_{\max }} \kappa_{i}(x) v(x) \mathrm{d} x .
\end{aligned}
$$

Assuming AQM and wireless packet losses to be independent from each other, the average packet loss probability for flow $i$ stemming from both ERD buffer management and wireless losses, denoted by $\overline{p_{i}}$, can be written as follows:

$$
\overline{p_{i}}=1-\left(1-\operatorname{PER}_{m, s}\right)\left(1-\overline{q_{i}}\right),
$$

where $\overline{q_{i}}$, average packet loss probability for flow $i$ stemming only from ERD, is obtained via queue averaging:

$$
\overline{q_{i}}=\frac{1}{\overline{\lambda_{i}}}\left(\int_{0^{+}}^{x_{\max }} p(x) \lambda_{i}(x) v(x) \mathrm{d} x\right) .
$$

Furthermore, let $\bar{D}_{i}$ denote the average queuing delay in the router seen by flow $i$. Then, we can write

$$
\bar{D}_{i}=\frac{1}{\overline{\kappa_{i}}}\left(\int_{0^{+}}^{x_{\max }} \frac{x}{r_{m}} \kappa_{i}(x) v(x) \mathrm{d} x\right) .
$$

Consequently,

$$
\sigma_{\mathrm{RTT}, i}^{2}=\frac{1}{\overline{\kappa_{i}}}\left(\int_{0^{+}}^{x_{\max }}\left(\frac{x}{r_{m}}-\bar{D}_{i}\right)^{2} \kappa_{i}(x) v(x) \mathrm{d} x+\bar{D}_{i}^{2} \kappa_{i}(0) V(0)\right) .
$$

If we know the workload-dependent intensity of packet arrivals $\lambda(x)$, then we can find the stationary density $v(x)$ and the atom at zero $V(0)$ with the algorithm outlined before. However, $\lambda(x)$ depends on $\mathrm{RTT}_{i}(x)$ and $T_{0, i}(x)$, the latter depending on $\sigma_{\mathrm{RTT}, i}$ for all $i$. Moreover, $\lambda(x)$ depends on $\overline{p_{i}}$. Recognizing that $\sigma_{\mathrm{RTT}, i}$ and $\overline{p_{i}}$ can be obtained provided $v(x)$ and $V(0)$ are available, we propose a fixed-point algorithm for obtaining the stationary density of the queue occupancy. For this purpose, we define $\sigma_{\mathrm{RTT}, i}^{(k)}$ and $\bar{p}_{i}{ }^{(k)}$ to denote the estimates for the corresponding quantities at iteration $k$. At iteration $k+1$, $\lambda(x)$ is obtained using $\sigma_{\mathrm{RTT}, i}^{(k)}$ and ${\overline{p_{i}}}^{(k)}$. Via the solution of the workload-dependent $M / D / 1$ queue with intensity function $\lambda(x)$, we obtain $v(x)$ and $V(0)$. The new values of $\sigma_{\mathrm{RTT}, i}$ and $\overline{p_{i}}$ are obtained at this iteration using the identities (19) and (16), respectively, but exponentially smoothed with smoothing parameters $\alpha_{1}$ and $\alpha_{2}$, respectively, to obtain $\sigma_{\mathrm{RTT}, i}^{(k+1)}$ and $\bar{p}_{i}^{(k+1)}$. Iterations continue until the following two conditions are simultaneously satisfied:

$$
\left|\sigma_{\mathrm{RTT}, i}^{(k+1)}-\sigma_{\mathrm{RTT}, i}^{(k)}\right| / \sigma_{\mathrm{RTT}, i}^{(k)}<\epsilon_{1},\left|{\overline{p_{i}}}^{(k+1)}-{\overline{p_{i}}}^{(k)}\right| /{\overline{p_{i}}}^{(k)}<\epsilon_{2},
$$

for some normalized tolerance parameters $\epsilon_{1}$ and $\epsilon_{2}$. For numerical experimentation with the fixed-point algorithm, we fix $\epsilon_{1}=\epsilon_{2}=0.01, \alpha_{1}=0.7$, and $\alpha_{2}=$ 0.9 . We finalize this section by expressing the aggregate TCP throughput, the key parameter to be studied in our numerical examples, as $\left(1-\mathrm{PER}_{m, s}\right) L \sum_{i=0}^{N-1} \overline{\kappa_{i}}$.

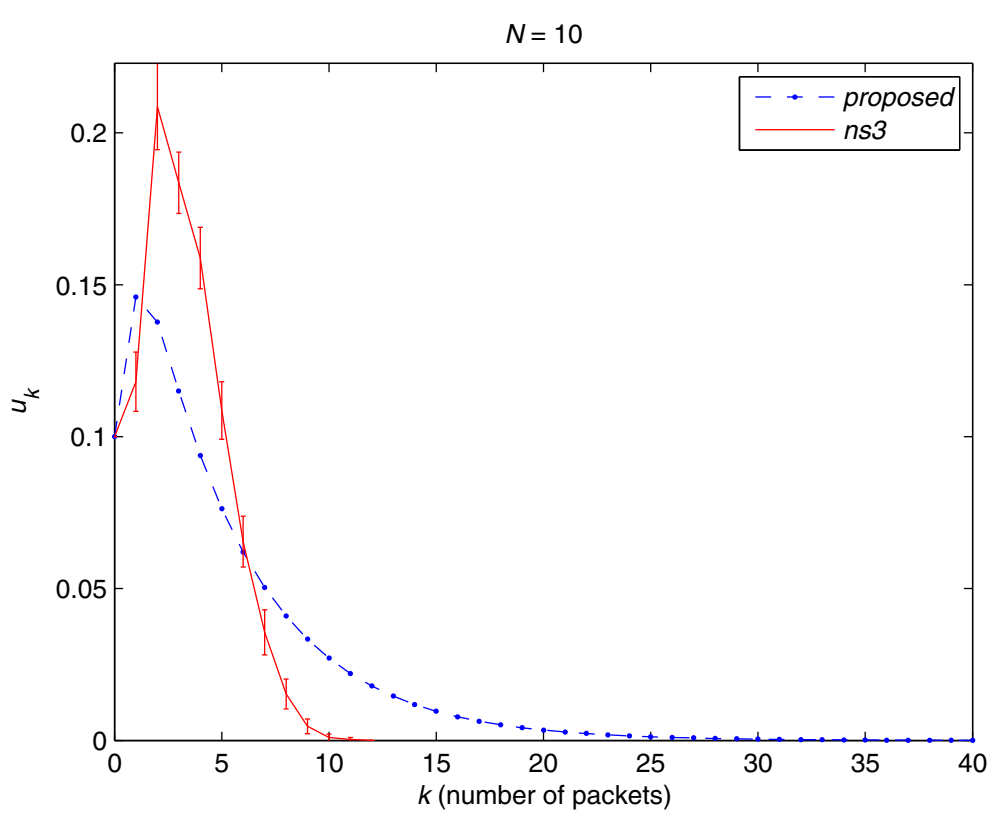

Figure 4 Queue occupancy PMF $u_{k}$ for scenario B with $N=10$ and $r_{L}=0.9$ Mbps. 


\section{Model validation}

The so-called proposed analysis method of this paper is validated using the ns-3 network simulator [22] for both wireline and wireless scenarios. We use the dumb-bell topology involving $N$ TCP Reno flows in Figure 1 in our simulations. The ingress link for flow $i, 0 \leq i<N$, has capacity $r_{R}$ and one-way propagation delay $D_{R_{i}}$, whereas the egress link for the same flow has capacity $r_{L}$ and one-way propagation delay $D_{L}$. The central link in the middle is the wireless bottleneck link with one-way propagation delay $D_{F}$ using an $\mathrm{MCS}_{m}$ yielding a capacity $r_{m}$ and a wireless loss rate $\mathrm{PER}_{m, s}$ at SNR level $\mathrm{SNR}_{s}$. TCP flow statistics are obtained using the FlowMonitor which is a monitoring framework developed for ns-3 [36]. The RateErrorModel class of ns-3 is used whenever a non-zero $\mathrm{PER}_{m, s}$ is to be simulated. In ns-3, it is more appropriate to probe queue occupancy in units of packets as opposed to the unfinished work density $v(x)$ obtained through the workload-dependent M/G/1 queuing model. Therefore, for the sake of comparing our results to those obtained by ns-3, we approximate the steady-state queue occupancy probability mass function (PMF) in units of packets denoted by $u_{k}$ as follows:

$u_{k}= \begin{cases}\sum_{i=1}^{\left\lceil x_{\max } / L\right\rceil}\left(\int_{y=((i-1) L)^{+}}^{i L} v(x) \mathrm{d} x\right) \delta(k-i), & k>0 \\ V(0), & k=0\end{cases}$

where $\delta(\cdot)$ denotes the Dirac-delta function. Simulations are terminated after $5 \mathrm{~min}$, but the first $30 \mathrm{~s}$ corresponding to transients are ignored. Each simulation is repeated ten times unless otherwise stated, and the average results are reported together with the associated confidence intervals computed for $95 \%$ confidence level.
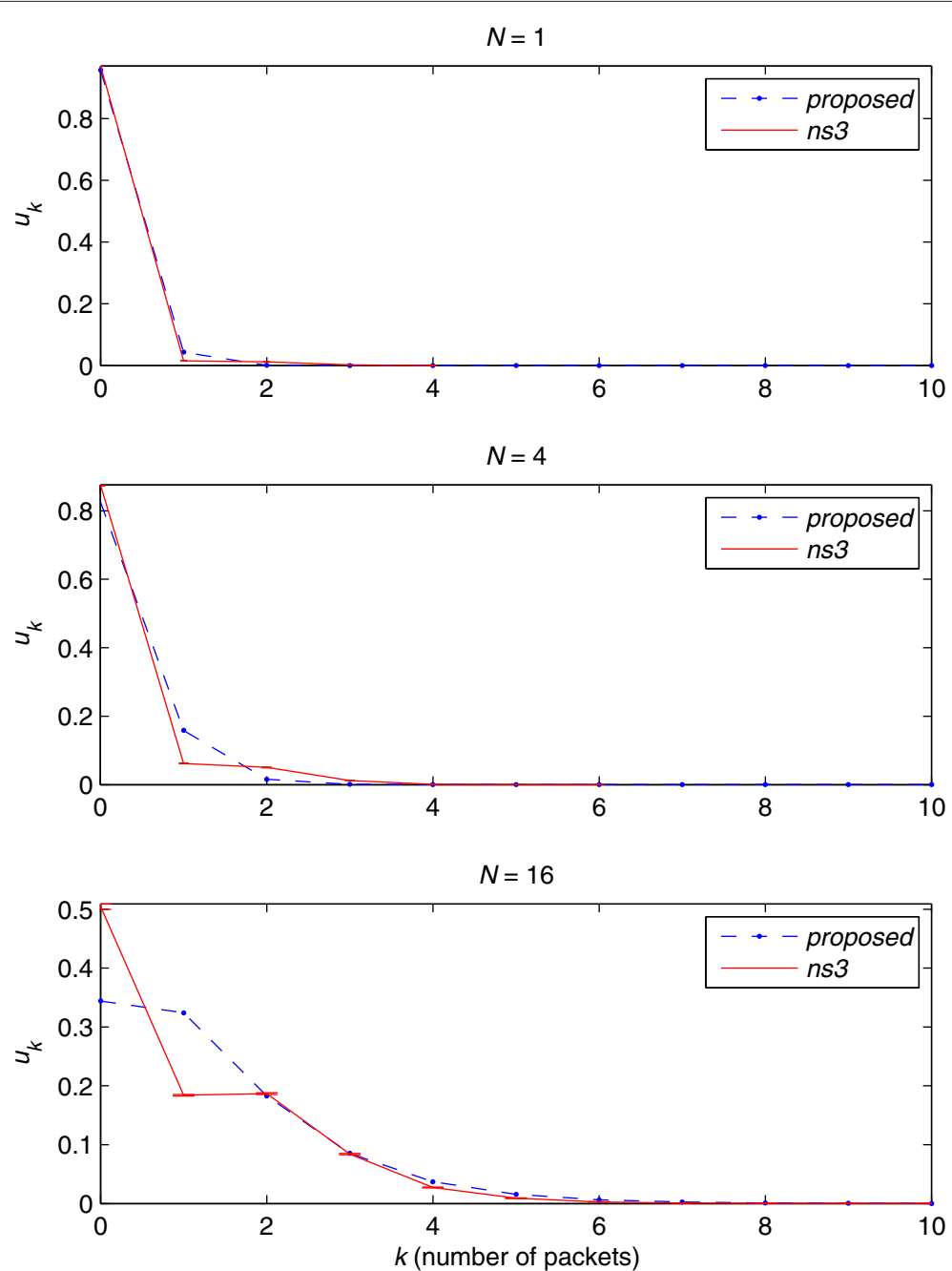

Figure 5 Queue occupancy PMF $u_{k}$ for scenario $C:\left(D_{L}, D_{F}, D_{R_{i}}\right)=(5,5,10)$ ms with PER $R_{m, s}=0.1$ for three different $N$ values. 
We compare the proposed analysis method with the one presented in [11] which is referred to as the fixedpoint method for the wireline scenario for which we set $\mathrm{PER}_{m, s}=0$. The method fixed-point pursues a similar approach to the proposed in relating the TCP throughput to the queue occupancy by the PFTK formula, but it uses a fluid model to obtain only the average queue occupancy, not its distribution. Moreover, fixed-point does not take wireless losses into account. We set $\left(r_{L}, r_{m}, r_{R}\right)=$ $(1,000,10,1,000) \mathrm{Mbps}$, ensuring that the central link is the bottleneck link. In Figure 2, the PMF $u_{k}$ is obtained using proposed, fixed-point, and ns-3 simulations for scenario A which refers to $\left(D_{L}, D_{F}, D_{R_{i}}\right)=(5,0,5) \mathrm{ms}$ and depicted for three different values of $N$. In Figure 3, the PMF $u_{k}$ is depicted for scenario $\mathrm{B}$ which refers to $\left(D_{L}, D_{F}, D_{R_{i}}\right)=(15,0,5+5\lfloor i /(N / 10)\rfloor) \mathrm{ms}$ again for three values of $N$. It is clear that the method proposed matches the PMF obtained by ns-3 simulations in both shape and magnitude especially for smaller number of flows. The deviation from the simulation results for larger number of flows is probably due to the fact that the instantaneous queuing delay is used in our workload-dependent queuing model although in actual TCP implementations, this information would be slightly delayed. Consequently, proposed improves upon fixed-point as far as the queue occupancy PMF is concerned.

For scenario B, we now set $N=10$ and $r_{L}=0.9 \mathrm{Mbps}$ (corresponding to $\lambda_{i, \max }=r_{L} / L$ of proposed), for which the numerical results are given in Figure 4. We also increase the number of ns-3 simulations to 120 to obtain more reasonable confidence intervals. In this case, fixedpoint ends up with an always-empty queue, whereas the proposed captures the actual PMF acceptably well. In particular, the probability mass at zero $V(0)$ is found to be $0.1000188,1$, and 0.0999987 , using proposed, fixed-point, and simulations, respectively. In a network of queues, it is likely that most of the links will be operating at an empty queue regime. We believe that the PMF-capturing
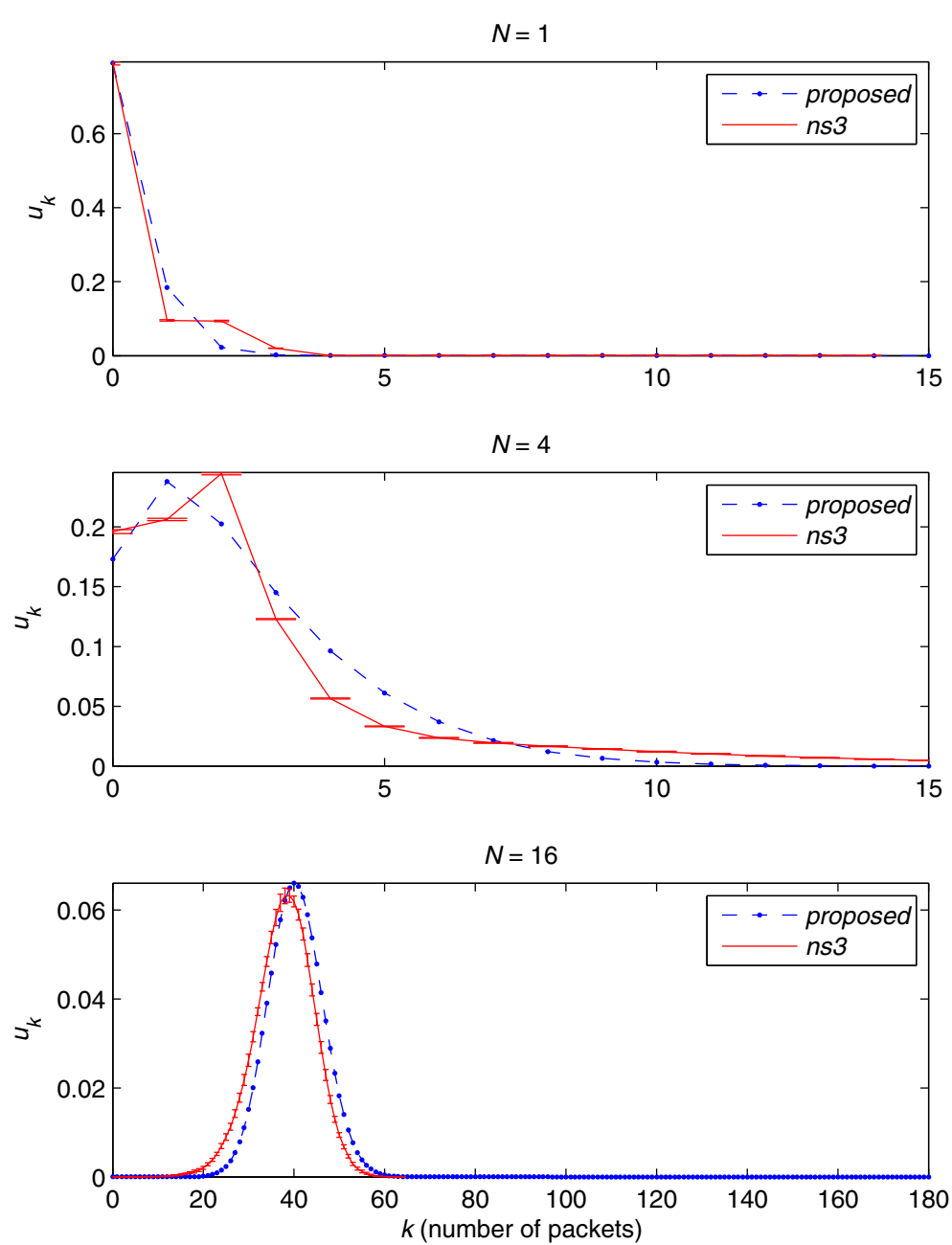

Figure 6 Queue occupancy PMF $u_{k}$ for scenario $C:\left(D_{L}, D_{F}, D_{R_{i}}\right)=(5,5,10) \mathrm{ms}$ with PER $\mathrm{R}_{m, s}=0.01$ for three different $N$ values. 
capability of proposed in the empty-queue regime will be crucial in the analysis of such systems.

For the final validation example in the wireless context, we study scenario $\mathrm{C}$ which refers to $\left(D_{L}, D_{F}, D_{R_{i}}\right)=$ $(5,5,10) \mathrm{ms}$ employing non-zero $\mathrm{PER}_{m, s}$. Queue occupancy PMF results using proposed and simulations are provided in Figures 5, 6, and 7 for $\mathrm{PER}_{m, s}$ being equal to $0.1,0.01$, and 0.001 , respectively, each for three values of $N$, i.e., $N=1,4,16$. For $N=4$ and $\mathrm{PER}_{m, s}=0.01$ in Figure 6 , the number of simulations is again set to 120 in order to increase the reliability of simulations. The simulation PMF appears to be captured well by the proposed analysis method for a wide range of packet error rates. For $N=1$ and $\mathrm{PER}_{m, s}=0.001$, ns-3 simulations show a peak which is caused by alternating on and off times during which the queue is on and off, respectively, and the Poisson assumption does not hold as well as the other cases. However, the general shape of the PMF is still captured for this challenging scenario.

\section{Cross-layer framework}

The physical layer Wireless-MAN OFDMA PHY specifies a cellular system comprising a base station (BS) and attached mobile stations (MSs) [25]. In this section, we perform cross-layer analysis of the IEEE 802.16 WirelessMAN OFDMA PHY air interface which can also alternatively be used for a point-to-point (PTP) wireless link $[37,38]$. We study this wireless link carrying long-lived TCP traffic flows for different values of $N, \mathrm{RTT}_{0, i}$, and $\mathrm{SNR}_{s}$ and for two different wireless channel models. This analysis suits well to the OFDM-based air interfaces which became viable for PTP wireless links $[39,40]$ while also being applicable to the downlink of a cellular system with FIFO queuing.

We run physical layer simulations with Coded Modulation Library (CML) to obtain the $\mathrm{PER}_{m, s}$ values for given $\mathrm{MCS}_{m}$ and $\mathrm{SNR}_{s}$ [41]. For this purpose, we choose the MCSs that use convolutional turbo codes (CTC). There exist 32 MCSs for CTC out of which $M=8$ are
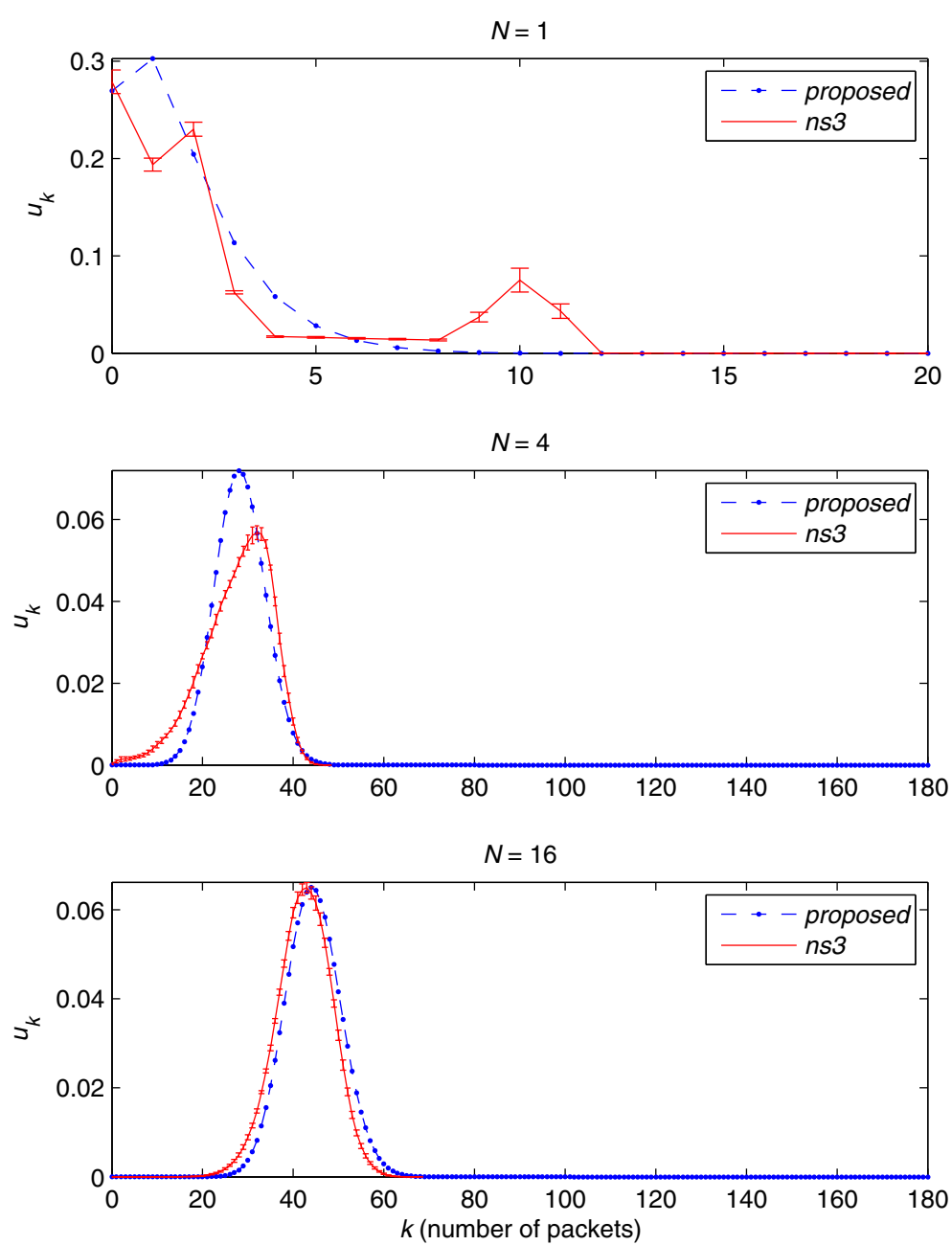

Figure 7 Queue occupancy PMF $u_{k}$ for scenario $C:\left(D_{L}, D_{F}, D_{R_{i}}\right)=(5,5,10)$ ms with PER ${ }_{m, s}=0.001$ for three different $N$ values. 
Table 1 Modulation and coding schemes of IEEE 802.16 to be used in this study

\begin{tabular}{lccc}
\hline $\boldsymbol{m}$ & $\boldsymbol{V}_{\boldsymbol{m}}$ & $\boldsymbol{R}_{\boldsymbol{m}}$ & $\boldsymbol{k}_{\boldsymbol{m}}$ (bytes) \\
\hline 0 & 4 & $1 / 2$ & 60 \\
1 & 4 & $3 / 4$ & 54 \\
2 & 16 & $1 / 2$ & 60 \\
3 & 16 & $3 / 4$ & 54 \\
4 & 64 & $1 / 2$ & 54 \\
5 & 64 & $2 / 3$ & 48 \\
6 & 64 & $3 / 4$ & 54 \\
7 & 64 & $5 / 6$ & 60 \\
\hline
\end{tabular}

enumerated in Table 1 for use in the current paper which differ according to their modulation order $V_{m}$ (i.e., the number of points in the constellation diagram), code rate $R_{m}$, and forward error correction (FEC) block length $k_{m}$. Assuming FEC block error events of a packet to be iid Bernoulli distributed, $\mathrm{PER}_{m, s}$ can be derived from the FEC block error rate $\mathrm{FER}_{m, s}$ as follows:

$$
\operatorname{PER}_{m, s}=1-\left(1-\mathrm{FER}_{m, s}\right)^{\left\lceil L / k_{m}\right\rceil} .
$$

On the other hand, the $\mathrm{FER}_{m, s}$ values in (22) can be obtained using CML. For the sake of completeness, we present all $\mathrm{FER}_{m, s}$ vs $\mathrm{SNR}_{s}$ curves in Figures 8 and 9 for the additive white Gaussian noise (AWGN) and International Telecommunication Union (ITU) Vehicular-A channels, respectively, the latter corresponding to an MS with a velocity of $90 \mathrm{~km} / \mathrm{h}$, which is referred to as ITU-A channel for the rest of the paper [42]. SNR ranges of [0 dB, 22 dB] and $[0 \mathrm{~dB}, 40 \mathrm{~dB}]$ are sampled with a resolution of 0.5 and $2 \mathrm{~dB}$ to obtain the corresponding $\mathrm{SNR}_{s}$ values for AWGN and ITU-A channels, respectively. At least $10^{7} \mathrm{FEC}$ blocks are decoded to reach the FEC block error rates shown. Once the $\mathrm{PER}_{m, s}$ values are obtained, they are fed into the workload-dependent $\mathrm{M} / \mathrm{D} / 1$ queuing model to estimate the aggregate TCP throughput for given $\mathrm{MCS}_{m}$ and $\mathrm{SNR}_{s}$.

For the sake of fairness in throughput comparisons between different MCSs, $L$ in (22) is set to the least common multiple of the $k_{m}$ values for $m \in\{0, . ., M-1\}$, which is equal to 2,160 bytes to avoid padding for any $\mathrm{MCS}_{m}$. Discretization interval $\Delta$ of the queue is set to 9.6 bits for this section which amounts to the packet size $L$ divided by 1,800. Time division duplex (TDD) mode as specified by WiMAX [43] dictates 35 downlink (DL) OFDM symbols, each having 768 data subcarriers for a channel bandwidth of $10 \mathrm{MHz}$ [25] within a TDD frame duration of $5 \mathrm{~ms}$, resulting in an average PHY rate of $r=5.376 \times 10^{6}$ subcarriers/s. Based on these parameters, the raw bit rate $r_{m}$ of the IEEE 802.16 Wireless-MAN OFDMA PHY air interface can be calculated in bps as follows:

$$
r_{m}=r \log _{2}\left(V_{m}\right) R_{m}
$$

In order to account for transmission, framing, and processing delays of the system, $D_{T}+2 D_{F}$ is set to $5 \mathrm{~ms}$. For the remaining set of parameters, we study scenarios for both AWGN and ITU-A channels spanning a wide range of $N, \mathrm{RTT}_{0, i}$, and $\mathrm{SNR}_{s}$ values where $s \in\left\{s_{\min }, . ., S-1\right\}$ and $s_{\min }$ is such that $\mathrm{PER}_{0, s_{\min }}<0.1$ for each channel type. In particular, we study two groups of scenarios having fixed and uniformly spaced $\mathrm{RTT}_{0, i}$ denoted by $\mathrm{SF}_{N, F}$

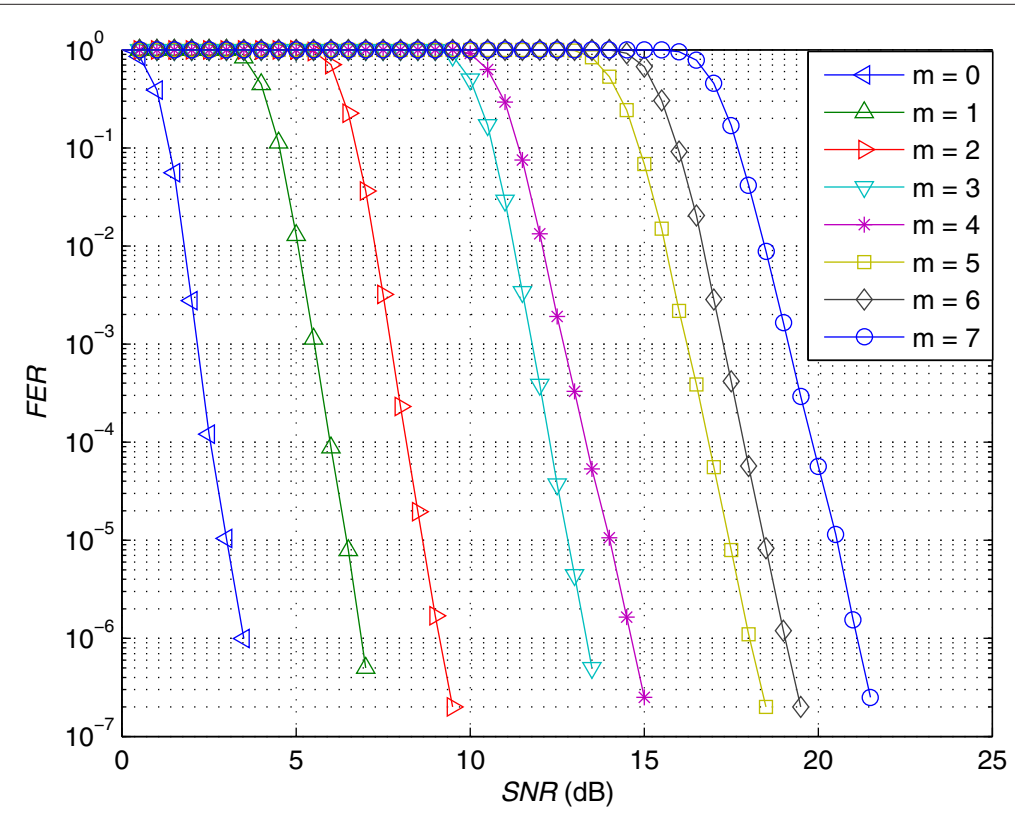

Figure 8 Simulated FEC block error rates (FER), AWGN channel. 


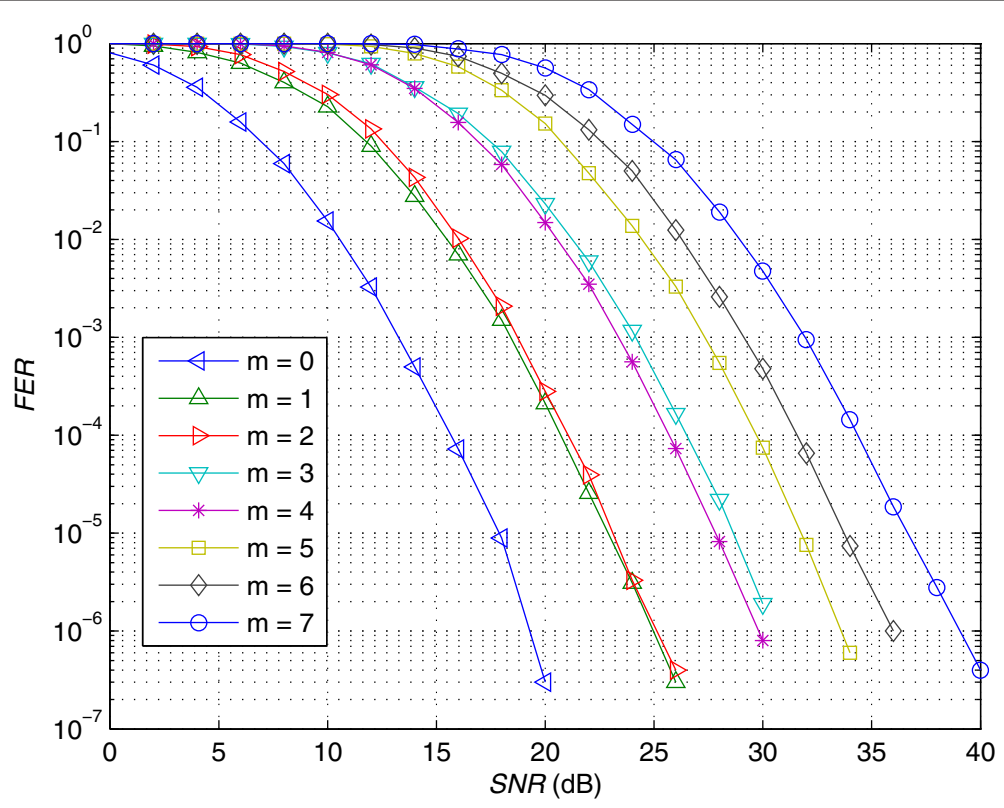

Figure 9 Simulated FEC block error rates (FER), ITU-A channel.

and $\mathrm{SU}_{N, F}$, respectively, where $N \in\{1,4,16\}$ and $F \in$ $\{1,5,10,20,40,80,120,160\} \mathrm{ms}$. In scenario $\mathrm{SF}_{N, F}$, there are $N$ long-lived TCP flows and all flows have the same $\mathrm{RTT}_{0, i}$ of $F \mathrm{~ms}$. On the other hand, in scenario $\mathrm{SU}_{N, F}$, there are again $N$ flows but each with a different $\mathrm{RTT}_{0, i}$. For $N=4$, flow $i, 0 \leq i \leq 3$, has its $\mathrm{RTT}_{0, i}$ set to $2(i+1) F / 5$, leading to an average fixed RTT of $F$ ms. For example, in $\mathrm{SU}_{4,10}$, flows have individual fixed RTT values of 4, 8, 12, and $16 \mathrm{~ms}$, with an average of $10 \mathrm{~ms}$. For $N=16$, each $\mathrm{RTT}_{0, i}$ value for the $N=4$ case is further mapped to four different fixed RTTs in a similar fashion to obtain the same average fixed RTT of $F$ ms. For example, in $\mathrm{SU}_{16,10}$, flows have individual fixed RTT values of $1.6,3.2,4.8,6.4,3.2,6.4,9.6,12.8,4.8,9.6,14.4,19.2,6.4$,

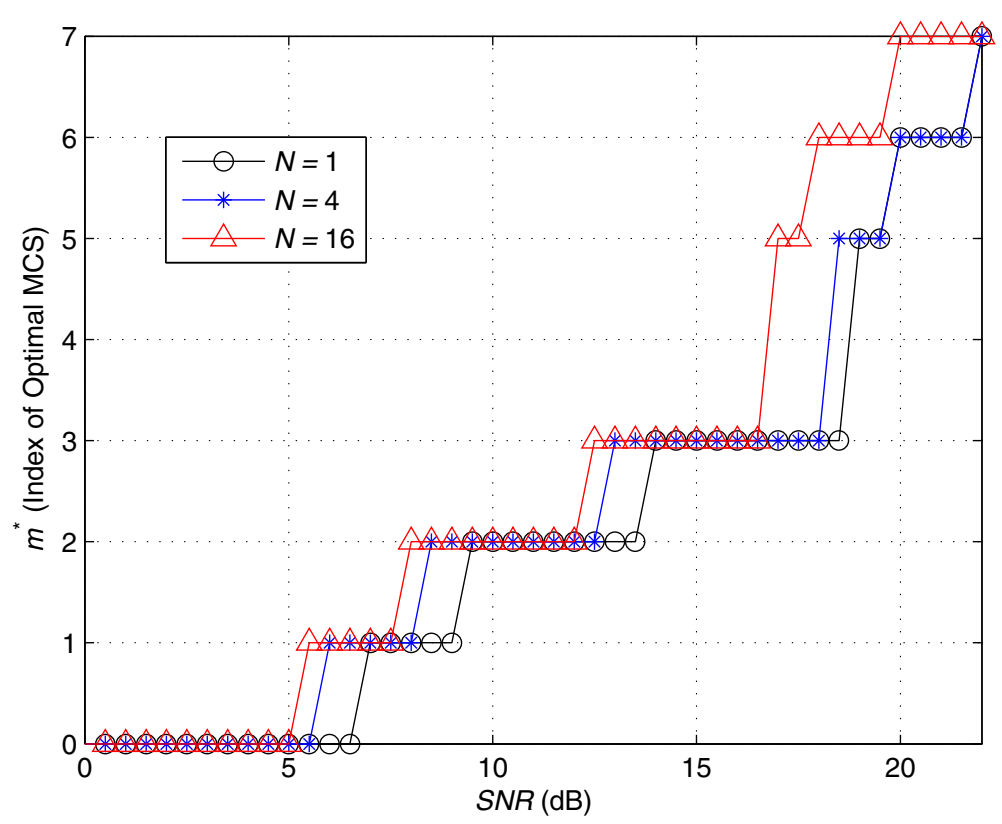

Figure 10 Optimal MCS index. Optimum MCS selection for AWGN channel as a function of SNR level SNR for scenarios $\mathrm{SF}_{1,160,} \mathrm{SF}_{4,160}$, and $\mathrm{SF}_{16,160}$ corresponding to $\mathrm{RT}_{0, i}=160 \mathrm{~ms}$ and $N=1, N=4$, and $N=16$, respectively. 


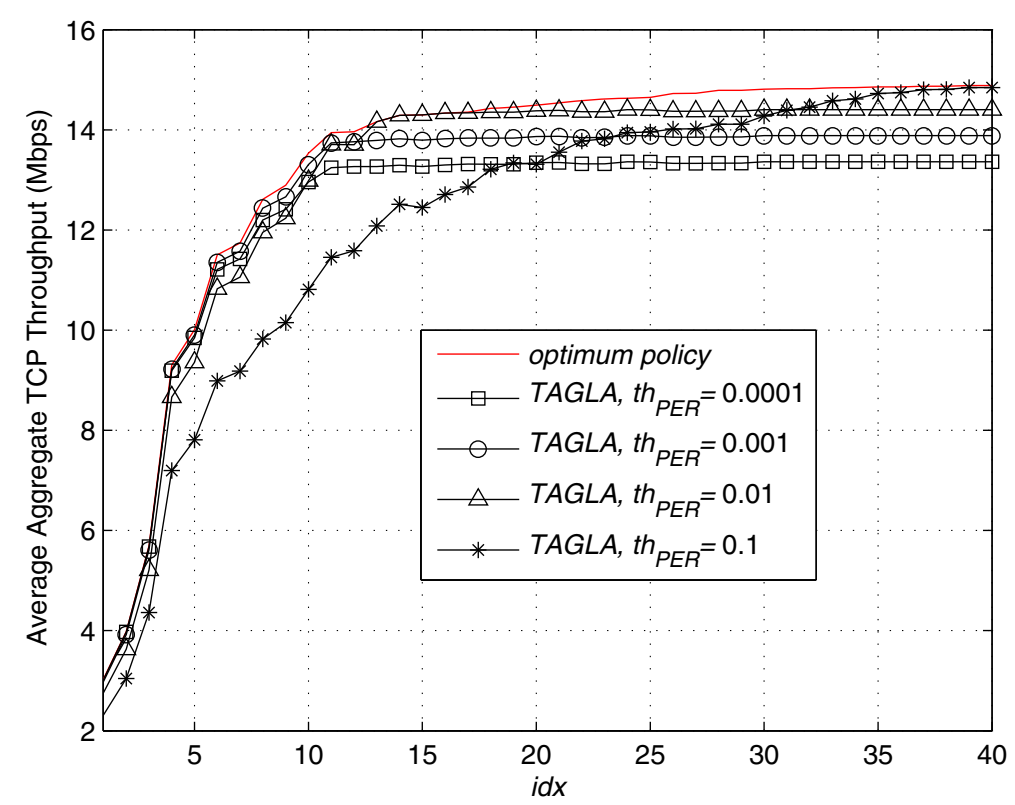

Figure 11 Average aggregate TCP throughput. This is achieved by the optimum policy and TAGLA averaged over SNR $\mathrm{f}_{s}$ for each scenario of Table 2 for the AWGN channel.

$12.8,19.2$, and $25.6 \mathrm{~ms}$, again with an average of $10 \mathrm{~ms}$. Note that scenarios represented by $\mathrm{SF}_{1, F}$ and $\mathrm{SU}_{1, F}$ are identical, thus leading to an overall of 40 unique scenarios.

In the first example, we study the particular scenarios $\mathrm{SF}_{N, 160}$ for different values of $N$ by solving the proposed queuing model and calculating the aggregate TCP throughput for each $\mathrm{MCS}_{m}, N$, and $\mathrm{SNR}_{s}$ for the AWGN channel. We present the optimal MCS index, denoted by $m^{*}$, leading to the highest aggregate TCP throughput in Figure 10 as a function of the channel SNR for all values of $N$. We observe that as $N$ increases, the benefit of increasing the spectral efficiency by choosing a higher order MCS outweighs the penalty of increasing wireless loss rate at certain SNR values. It is therefore clear that

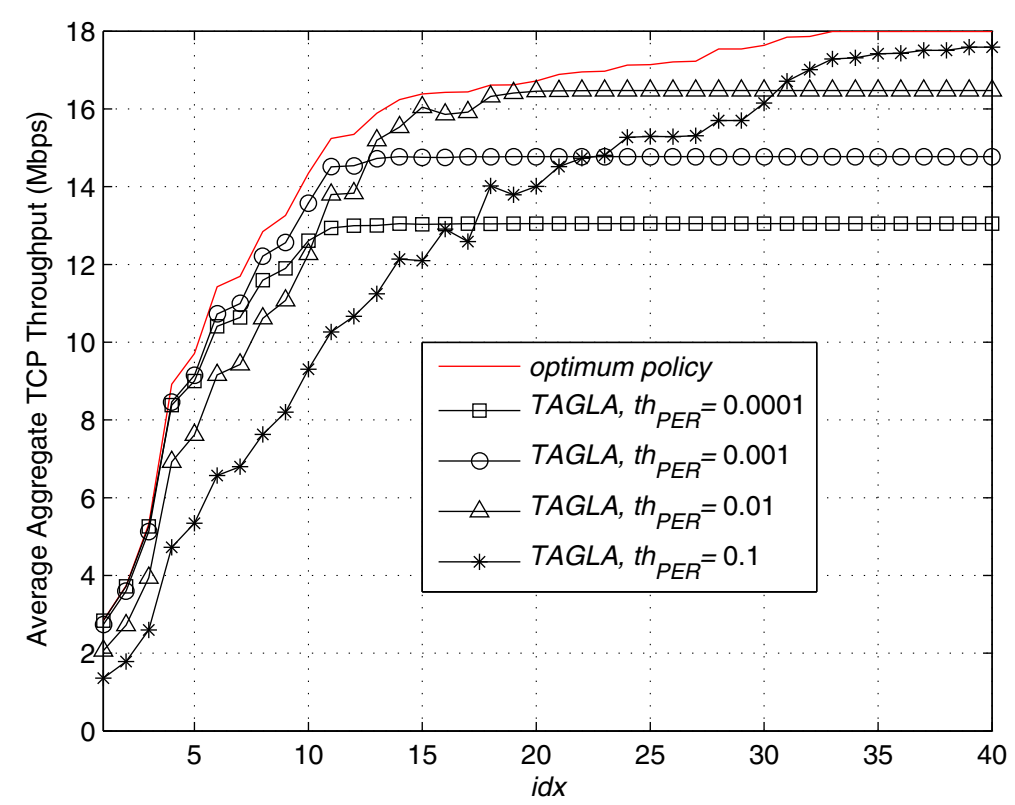

Figure 12 Average aggregate TCP throughput. This is achieved by the optimum policy and TAGLA averaged over SNR for $_{5}$ each scenario of Table 3 for the ITU-A channel. 
the optimal decision on MCS relies on a priori knowledge on the number of TCP flows sharing the link. Obtaining the optimum MCS in an online setting requires the estimation of $N$ and $\mathrm{RTT}_{0, i}$ which is generally known to be difficult. As a remedy, we propose the so-called trafficagnostic link adaptation (TAGLA) scheme which does not require the estimation of traffic parameters but takes into consideration only the spectral efficiency and FER values of the MCSs that its physical layer has to offer. In particular, TAGLA chooses the MCS with the highest spectral efficiency whose resulting $\mathrm{PER}_{m, s} \leq t h_{\mathrm{PER}}$ for a threshold parameter $t h_{\text {PER }}$. In case of plurality of such MCSs with the same spectral efficiency, TAGLA chooses the one with the lowest PER. At the limiting cases $t h_{\mathrm{PER}}=0$ and $t h_{\text {PER }}=1$, TAGLA resorts to $M C S_{0}$ and $M C S_{7}$, respectively, regardless of the $\mathrm{SNR}_{s}$ value. The TAGLA scheme only requires a mapping between the target PER values and the FER values to be continuously fed back by the PHY layer. Choice of the target PER parameter $t h_{\mathrm{PER}}$ is then crucial for the performance of the TAGLA scheme which we now study.

Averaging the results of the proposed model over all possible SNR values we study, the average aggregate TCP throughput as a function of these 40 scenarios is depicted in Figures 11 and 12 for various values of the $t h_{\text {PER }}$ parameter and for both channel models. The optimum aggregate TCP throughput obtained by choosing the best possible MCS, given $\mathrm{SNR}_{s}, N$, and $\mathrm{RTT}_{0, i}$, is also averaged in the same way and presented as a benchmark. The scenarios are indexed with a parameter called $i d x$ as shown in Tables 2 and 3, for increasing throughput of the so-called optimum policy in AWGN and ITU-A channels, respectively. Low values of $i d x$, corresponding to relatively low values for $N$ and large values for $\mathrm{RTT}_{0, i}$, are representative of situations in which the TCP flows cannot keep the queue always full. In such cases, the throughput is lower and the penalty of using larger values of $t h_{\mathrm{PER}}$ is apparent. Conversely, large values for $i d x$ are indicative of a situation in which TCP can keep the queue full all the time despite wireless losses, and the optimum policy is to use the MCS with the best spectral efficiency but with larger wireless loss rates. This observation remains intact for both channel models.

In order to assess the sensitivity of throughput to $t_{\text {PER }}$ in different scenario settings, we form two groups among the scenarios studied so far, namely $G_{\text {low }}$ and $G_{\text {high }}$, by partitioning the range of $i d x$ into subsets $[1, \ldots, 15]$ and $[26, . ., 40]$, respectively. We also let $G_{\text {all }}$ represent the group of all scenarios with $i d x \in[1, . ., 40]$. First, we normalize the throughput of TAGLA with that of optimum
Table 2 Scenarios $\mathrm{SF}_{N, F}$ and $\mathrm{SU}_{N, F}$ indexed by increasing throughput of optimum policy for the AWGN channel

\begin{tabular}{llll}
\hline $\boldsymbol{i d} \boldsymbol{x}$ & Scenario $_{1}$ & $\boldsymbol{i d \boldsymbol { x }}$ & $\mathrm{Scenario}$ \\
\hline 1 & $\mathrm{SF}_{1,160}$ & 21 & $\mathrm{SU}_{16,160}$ \\
2 & $\mathrm{SF}_{1,120}$ & 22 & $\mathrm{SF}_{4,10}$ \\
3 & $\mathrm{SF}_{1,80}$ & 24 & $\mathrm{SU}_{4,10}$ \\
4 & $\mathrm{SF}_{1,40}$ & $\mathrm{SF}_{16,80}$ \\
5 & $\mathrm{SF}_{4,160}$ & 25 & $\mathrm{SU}_{16,120}$ \\
6 & $\mathrm{SU}_{4,160}$ & 26 & $\mathrm{SF}_{4,5}$ \\
7 & $\mathrm{SF}_{4,120}$ & 27 & $\mathrm{SU}_{4,5}$ \\
8 & $\mathrm{SF}_{1,20}$ & 28 & $\mathrm{SF}_{4,1}$ \\
9 & $\mathrm{SU}_{4,120}$ & 29 & $\mathrm{SU}_{4,1}$ \\
10 & $\mathrm{SF}_{4,80}$ & 30 & $\mathrm{SU}_{16,80}$ \\
11 & $\mathrm{SF}_{1,10}$ & 31 & $\mathrm{SF}_{16,40}$ \\
12 & $\mathrm{SU}_{4,80}$ & 32 & $\mathrm{SU}_{16,40}$ \\
13 & $\mathrm{SF}_{1,5}$ & 33 & $\mathrm{SF}_{16,20}$ \\
14 & $\mathrm{SF}_{4,40}$ & 34 & $\mathrm{SU}_{16,20}$ \\
15 & $\mathrm{SF}_{1,1}$ & 35 & $\mathrm{SF}_{16,10}$ \\
16 & $\mathrm{SU}_{4,40}$ & 36 & $\mathrm{SU}_{16,10}$ \\
17 & $\mathrm{SF}_{16,160}$ & 37 & $\mathrm{SF}_{16,5}$ \\
18 & $\mathrm{SF}_{4,20}$ & 38 & $\mathrm{SU}_{16,5}$ \\
19 & $\mathrm{SU}_{4,20}$ & 39 & $\mathrm{SF}_{16,1}$ \\
20 & $\mathrm{SF}_{16,120}$ & 40 & $\mathrm{SU}_{16,1}$ \\
\hline
\end{tabular}

Table 3 Scenarios $\mathrm{SF}_{N, F}$ and $\mathrm{SU}_{N, F}$ indexed by increasing throughput of optimum policy for the ITU-A channel

\begin{tabular}{llll}
\hline $\boldsymbol{i d \boldsymbol { x }}$ & $\mathrm{Scenario}_{1,160}$ & $\boldsymbol{i d \boldsymbol { x }}$ & Scenario \\
\hline 1 & $\mathrm{SF}_{1,160}$ & 21 & $\mathrm{SU}_{16,160}$ \\
2 & $\mathrm{SF}_{1,120}$ & 22 & $\mathrm{SF}_{4,10}$ \\
3 & $\mathrm{SF}_{1,80}$ & 23 & $\mathrm{SU}_{4,10}$ \\
4 & $\mathrm{SF}_{1,40}$ & 24 & $\mathrm{SU}_{16,120}$ \\
5 & $\mathrm{SF}_{4,160}$ & 25 & $\mathrm{SF}_{16,80}$ \\
6 & $\mathrm{SU}_{4,160}$ & 26 & $\mathrm{SF}_{4,5}$ \\
7 & $\mathrm{SF}_{4,120}$ & 27 & $\mathrm{SU}_{4,5}$ \\
8 & $\mathrm{SF}_{1,20}$ & 28 & $\mathrm{SF}_{4,1}$ \\
9 & $\mathrm{SU}_{4,120}$ & 29 & $\mathrm{SU}_{4,1}$ \\
10 & $\mathrm{SF}_{4,80}$ & 30 & $\mathrm{SU}_{16,80}$ \\
11 & $\mathrm{SF}_{1,10}$ & 31 & $\mathrm{SF}_{16,40}$ \\
12 & $\mathrm{SU}_{4,80}$ & 32 & $\mathrm{SU}_{16,40}$ \\
13 & $\mathrm{SF}_{1,5}$ & 33 & $\mathrm{SF}_{16,20}$ \\
14 & $\mathrm{SF}_{4,40}$ & 34 & $\mathrm{SU}_{16,20}$ \\
15 & $\mathrm{SF}_{1,1}$ & 35 & $\mathrm{SF}_{16,10}$ \\
16 & $\mathrm{SF}_{16,160}$ & 36 & $\mathrm{SU}_{16,10}$ \\
17 & $\mathrm{SU}_{4,40}$ & 37 & $\mathrm{SF}_{16,5}$ \\
18 & $\mathrm{SF}_{16,120}$ & 38 & $\mathrm{SU}_{16,5}$ \\
19 & $\mathrm{SF}_{4,20}$ & 39 & $\mathrm{SF}_{16,1}$ \\
20 & $\mathrm{SU}_{4,20}$ & 40 & $\mathrm{SU}_{16,1}$ \\
\hline & & & \\
\hline & & 36 & 29 \\
\hline
\end{tabular}




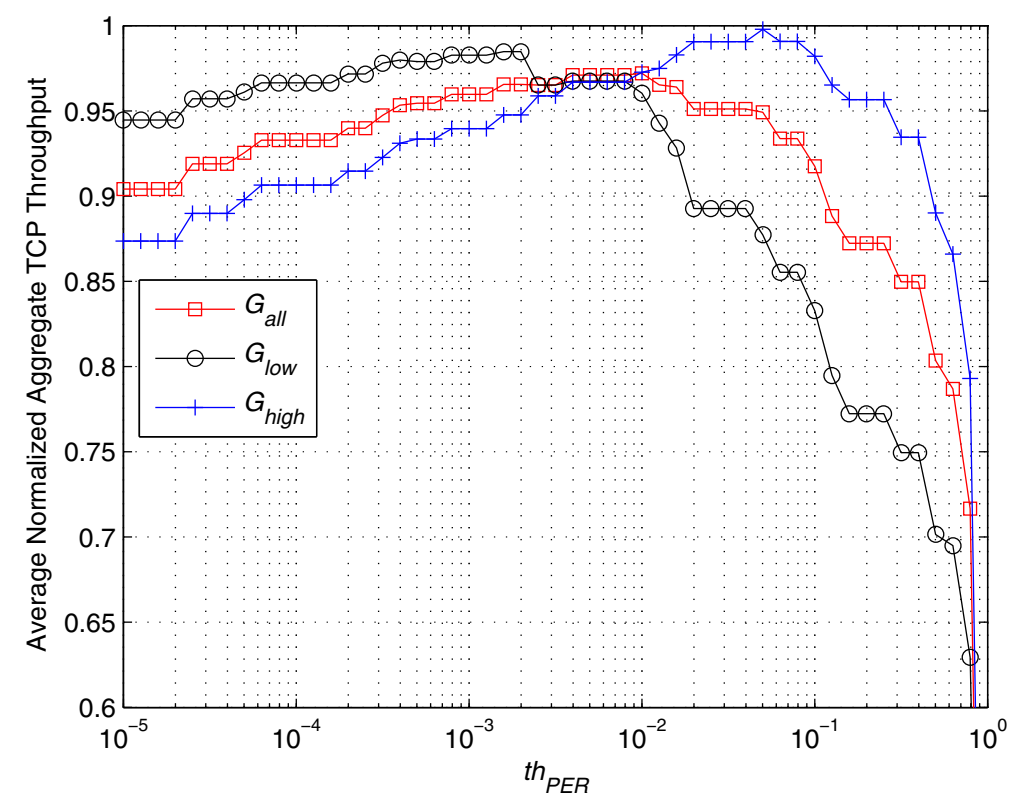

Figure 13 Average normalized aggregate TCP throughput. This is achieved by TAGLA averaged over SNRs for scenarios $G_{a l l}, G_{l o w}$, and Ghigh for the AWGN channel.

policy for each $\mathrm{SNR}_{s}$ and $i d x$ to avoid a bias favoring MCSs with high spectral efficiency. Then, we average the normalized throughput values over $\mathrm{SNR}_{s}$ and $i d x$ within the ranges of each group to obtain the average normalized aggregate TCP throughput shown in Figures 13 and 14 for AWGN and ITU-A channels, respectively. The choice of $t h_{\text {PER }}=5 \times 10^{-3}$ turns out to be a robust operating point for the three groups resulting in at most $4 \%$ and $12 \%$ performance penalties with respect to the optimum policy for AWGN and ITU-A channels, respectively. Targeting higher (lower) wireless loss rates has a negative impact on the TCP throughput especially for $G_{\text {low }}$

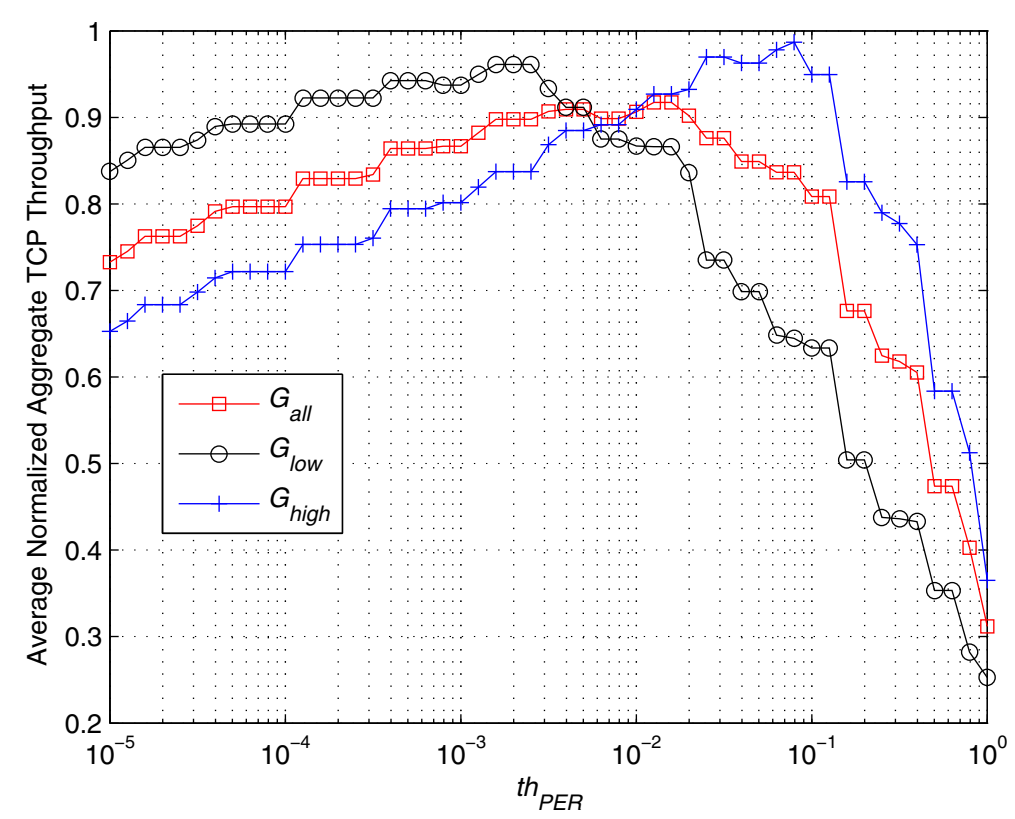

Figure 14 Average normalized aggregate TCP throughput. This is achieved by TAGLA averaged over SNRs for scenarios $G_{\text {alll }}, G_{l o w}$, and $G_{h i g h}$ for the ITU-A channel. 
$\left(G_{\text {high }}\right)$. The reduction in TCP throughput around the proposed $t h_{\text {PER }}$ value is more pronounced for the ITU-A channel which can be accounted for its relatively flat FER vs SNR curves shown in Figure 9. For a given SNR, FER values of the ITU-A channel are relatively closer to each other on the average. For this reason, the performance of TAGLA is more sensitive to the choice of $t h_{\text {PER }}$ for this multipath fading channel model. The TAGLA scheme appears to have a potential for improvement provided that the threshold parameter $t h_{\text {PER }}$ could be adaptively changed based on an estimation of the underlying traffic parameters which is left for future research.

\section{Conclusions}

In this study, we develop a novel workload-dependent queuing model for AQM-controlled wireless routers carrying persistent TCP flows. One of the contributions of this model is in its ability to capture the entire queue occupancy distribution as opposed to simpler performance measures of interest such as the mean queue length. The proposed queuing model is validated using ns-3 simulations in both wireline and wireless scenarios. This analytical method is then used to obtain guidelines for setting the target wireless packet error rate (PER) for a PERbased traffic-agnostic link adaptation scheme. Assuming wireless channel SNR to be uniformly distributed over the presented ranges of interest and packet losses to be concentrated on the wireless link (i.e., wireline losses are negligible), we show that targeting a PER around $5 \times 10^{-3}$ irrespective of the underlying traffic parameters provides robust and acceptable average TCP performance for a wide range of scenarios and for the given AQM setting. Future work will consist of the study of traffic-aware link adaptation policies and HARQ/ARQ techniques in more depth for TCP throughput optimization in wireless links.

\section{Competing interests}

The authors declare that they have no competing interests.

\section{Acknowledgments}

This work is supported in part by the Science and Research Council of Turkey (Tübitak) under project grant EEEAG-111E106.

Received: 26 October 2013 Accepted: 6 April 2014

Published: 27 April 2014

\section{References}

1. B Braden, D Clark, J Crowcroft, B Davie, S Deering, D Estrin, S Floyd, V Jacobson, G Minshall, C Partridge, L Peterson, K Ramakrishnan, S Shenker, J Wroclawski, L Zhang, Recommendations on queue management and congestion avoidance in the internet. RFC 2309 (1998). http://www.rfceditor.org/rfc/rfc2309.txt. Accessed 21 April 2014

2. S Floyd, $V$ Jacobson, Random early detection gateways for congestion avoidance. Netw. IEEE/ACM Trans. 1(4), 397-413 (1993)

3. ES Hashem, Analysis of random drop for gateway congestion control. MSc thesis, Massachusetts Inst of Tech Cambridge Lab for Computer Science, (1989)

4. S Athuraliya, SH Low, VH Li, Q Yin, REM: Active queue management. Netw. IEEE. 15(3), 48-53 (2001)
5. M Hassan, R Jain, High Performance TCP/IP Networking: Concepts, Issues, and Solutions. (Pearson, US, 2004)

6. R Adams, Active queue management: a survey. Commun. Surv. Tutorials, IEEE. 15(3), 1425-1476 (2013)

7. J Lakkakorpi, A Sayenko, J Karhula, O Alanen, J Moilanen, Active queue management for reducing downlink delays in WiMAX, in Vehicular Technology Conference, 2007. VTC-2007 Fall. 2007 IEEE 66th, Baltimore, MD, USA, September 2007, pp. 326-330

8. J Padhye, V Firoiu, D Towsley, J Kurose, Modeling TCP throughput: a simple model and its empirical validation. SIGCOMM Comput. Commun Rev. 28(4), 303-314 (1998)

9. M Mathis, J Semke, J Mahdavi, T Ott, The macroscopic behavior of the TCP congestion avoidance algorithm. Comput. Commun. Rev. 27(3), 67-82 (1997)

10. P Lassila, H van den Berg, M Mandjes, R Kooij, ed. by J Charzinski, R Lehnert, and P Tran-Gia, An integrated packet/flow model for TCP performance analysis, in Providing Quality of Service in Heterogeneous Environments. Teletraffic Science and Engineering, vol. 5a (Elsevier Amsterdam, 2003), pp. 651-660

11. T Bu, D Towsley, Fixed point approximations for TCP behavior in an AQM network. SIGMETRICS Perform. Eval. Rev. 29(1), 216-225 (2001)

12. RJ Gibbens, SK Sargood, CV Eijl, FP Kelly, H Azmoodeh, RN Macfadyen, NW Macfadyen, Fixed-point models for the end-to-end performance analysis of IP networks, in Proceedings of the 13th ITC Specialist Seminar: IP Traffic Measurement, Modeling and Management, Monterey, CA, USA, September 2000

13. H Balakrishnan, VN Padmanabhan, S Seshan, RH Katz, A comparison of mechanisms for improving TCP performance over wireless links. Netw. IEEE/ACM Trans. 5(6), 756-769 (1997)

14. C Barakat, E Altman, Bandwidth tradeoff between TCP and link-level FEC Comput. Netw. 39(2), 133-150 (2002)

15. F Vacirca, A De Vendictis, A Baiocchi, Optimal design of hybrid FEC/ARQ schemes for TCP over wireless links with Rayleigh fading. IEEE Trans. Mobile Comput. 5(4), 289-302 (2006)

16. K-C Go, J-R Cha, SK Oh, J-H Kim, End-to-end performance analysis based on cross-layer retransmission scheme in wireless communication system. Int. Conf. Inform. Netw. 2013 (ICOIN) 0, 141-144 (2013)

17. D Moltchanov, A study of TCP performance in wireless environment using fixed-point approximation. Comput. Netw. 56(4), 1263-1285 (2012)

18. S Catreux, V Erceg, D Gesbert, RW Heath Jr, Adaptive modulation and MIMO coding for broadband wireless data networks. Comm. Mag. 40(6), 108-115 (2002)

19. M Lampe, $H$ Rohling, W Zirwas, Misunderstandings about link adaptation for frequency selective fading channels, in Personal, Indoor and Mobile Radio Communications, 2002. The 13th IEEE International Symposium on vol. 2, Lisbon, Portugal, September 2002, pp. 710-714

20. M Lampe, T Giebel, H Rohling, W Zirwas, PER-prediction for PHY mode selection in OFDM communication systems, in IEEE Global Telecommunications Conference, GLOBECOM '03, San Francisco, CA, USA, December 2003, pp. 25-29

21. G Martorell, F Riera-Palou, G Femenias, Cross-layer fast link adaptation for MIMO-OFDM based WLANs. Wirel. Pers. Commun. 56(3), 599-609 (2011)

22. Ns-3. http://www.nsnam.org/. Accessed 21 April 2014

23. QoS: Congestion avoidance configuration guide, Cisco IOS release 15.0M. Technical report (Cisco Systems, Inc., San Jose, 2011)

24. M Wurtzler, Analysis and simulation of weighted random early detection (WRED) queues. Master's thesis. University of Kansas, 2003

25. IEEE standard for local and metropolitan area networks part 16: Air interface for broadband wireless access systems. IEEE Std 802.16-2009 (Revision of IEEE Std 802.16-2004), 1-2080 (2009)

26. M Laor, $L$ Gendel, The effect of packet reordering in a backbone link on application throughput. Netw. IEEE. 16(5), 28-36 (2002)

27. J Li, YQ Zhao, Resequencing analysis of stop-and-wait $A R Q$ for parallel multichannel communications. Netw. IEEE/ACM Trans. 17(3), 817-830 (2009)

28. R Bekker, SC Borst, OJ Boxma, O Kella, Queues with workload-dependent arrival and service rates. Queueing Syst. 46(3-4), 537-556 (2004)

29. V Vasudevan, A Phanishayee, H Shah, E Krevat, DG Andersen, GR Ganger, GA Gibson, B Mueller, Safe and effective fine-grained TCP retransmissions for datacenter communication. SIGCOMM Comput. Commun. Rev. 39(4), 303-314 (2009) 
30. H Abdel-jaber, F Thabtah, AM Daoud, J Ababneh, M Baklizi, Performance investigations of some active queue management techniques using simulation. Int. J. New Comput. Architectures Appl. (IJNCAA).

2(1), 286-301 (2012)

31. S Floyd, Recommendation on using the Gentle variant of RED. Technical report (The ICSI Networking and Security Group, March 2000)

32. E Kuumola, J Resing, J Virtamo, Joint distribution of instantaneous and averaged queue length in an $\mathrm{M} / \mathrm{M} / 1 / \mathrm{K}$ system, in Proceedings of the 15th ITC Specialist Seminar Internet Traffic Engineering and Traffic Management, Wurzburg, Germany, July 2002, pp. 58-67

33. A Misra, TJ Ott, The window distribution of idealized TCP congestion avoidance with variable packet loss, in INFOCOM '99. Eighteenth Annual Joint Conference of the IEEE Computer and Communications Societies. Proceedings. IEEE, vol. 3, New York, NY, USA, March 1999, pp. 1564-1572

34. H Balakrishnan, VN Padmanabhan, G Fairhurst, M Sooriyabandara, TCP performance implications of network path asymmetry. RFC 3449, RFC Editor (December 2002). http://www.rfc-editor.org/rfc/rfc3449.txt. Accessed 21 April 2014

35. TJ Speight, Efficient TCP ACK prioritization in wireless networks. US20080080464 A1, April 2008

36. G Carneiro, P Fortuna, M Ricardo, FlowMonitor: a network monitoring framework for the network simulator 3 (NS-3), in Proceedings of the Fourth International ICST Conference on Performance Evaluation Methodologies and Tools. VALUETOOLS '09, ICST (Institute for Computer Sciences, Social-Informatics and Telecommunications Engineering), Brussels, Belgium, 2009, pp. 1-1110

37. DT Chen, On the analysis of using 802.16e WiMAX for point-to-point wireless backhaul, in Radio and Wireless Symposium, IEEE, Long Beach, CA, USA, January 2007, pp. 507-510

38. JARP de Carvalho, H Veiga, P Gomes, AD Reis, R Costa, A contribution to experimental performance evaluation of point-to-point WiMAX links, in Signal Processing and Information Technology. ISSPIT 2008. IEEE International Symposium On, Sarajevo, December 2008, pp. 150-153

39. PTP solutions guide Motorola fixed point-to-point wireless bridges. http://www.motorolasolutions.com/. Accessed 21 April 2014

40. RDL-3000 System. http://rdlcom.com/. Accessed 21 April 2014

41. Coded Modulation Library (CML). http://www.terativesolutions.com/. Accessed 21 April 2014

42. Guidelines for evaluation of radio transmission technologies for IMT-2000. Recommendation ITU-R M.1225 (International Telecommunications Union (ITU), 1997)

43. WiMAX Forum mobile system profile: Release 1.5 TDD specific part. Technical report (WiMAX FORUM, March 2009)

doi:10.1186/1687-1499-2014-67

Cite this article as: Ozturk and Akar: Workload-dependent queuing model of an AQM-controlled wireless router with TCP traffic and its application to PER-based link adaptation. EURASIP Journal on Wireless Communications and Networking 2014 2014:67.

\section{Submit your manuscript to a SpringerOpen ${ }^{\circ}$ journal and benefit from:}

- Convenient online submission

- Rigorous peer review

- Immediate publication on acceptance

- Open access: articles freely available online

- High visibility within the field

- Retaining the copyright to your article

Submit your next manuscript at $\boldsymbol{\wedge}$ springeropen.com 